\title{
Infrared Microspectroscopy: How Diffraction Affects Spatial Resolution and Contrast
}

\author{
G.L. Carr, G.D. Smith and L.M. Miller \\ National Synchrotron Light Source, Brookhaven National Laboratory, Upton, NY 11973
}

Infrared microspectroscopy has evolved to become a standard method for spatially resolving the chemical and physical properties of heterogeneous materials. Under optimum conditions, the spatial resolution and contrast are determined by the diffraction of light. Though the blurring introduced by diffraction is familiar to all microscopists, its detailed effect on infrared microscopy has received little attention. An important difference between a visible light microscope and an infrared microscope is that the latter usually incorporates Schwarzschild-type reflecting objectives. These microscope objectives have an annular aperture rather than an open disk, so the diffraction pattern differs significantly from the common Airy pattern.

In this work, we present results from an investigation of the Schwarzschild objective's diffraction pattern and its impact on the performance of an imaging infrared microspectrometer. The calculated resolution and contrast for various test specimens are compared with experimental measurements. Infrared radiation from an electron synchrotron provides a high-brightness source that enables microspectroscopy to be performed at the diffraction-limit while still achieving high signal-to-noise $(\mathrm{S} / \mathrm{N})$. In general, the agreement between calculation and experiment is very good. This implies that for situations where the $\mathrm{S} / \mathrm{N}$ is very high, image deconvolution may become a practical method for increasing both the resolution and contrast for infrared imaging. As an example, the edge of a photoresist layer[1] was scanned at a wavelength of $6 \mu \mathrm{m}$ and yielded the absorption profile shown in figure 1. Using a $10 \%$ to $90 \%$ criteria, the edge appears more than $12 \mu \mathrm{m}$ wide. When the diffraction pattern appropriate to the microscope's Schwarzschild objective is deconvolved from this profile, the apparent edge width is reduced by almost of factor of 3 . We have also studied other test specimens, such as samples made up of circular disks and circular holes. In some situations, diffraction artifacts are predicted to appear and subsequently observed in actual measurements. An example is illustrated in figure 2 , which shows the measured absorption for $\lambda=6 \mu \mathrm{m}$ as a function of position when the microspectrometer beam is scanned across a $12 \mu \mathrm{m}$ diameter empty hole in a layer of photoresist. As a result of diffraction, the absorption inside the hole never falls below 50\%, plus a distinct "bump" appears near the middle of the hole. Both are accurately predicted by the calculation. Diffraction calculations also indicate that confocal apertures should significantly reduce many of these artifacts. Lastly, we consider other commonly used infrared microscope objectives having even more restrictive apertures, such as the ones used for grazing incidence and attenuated total reflectance (ATR).

[1] Resolution test specimen courtesy of John Reffner, SensIR Inc.

[2] Research supported by the U.S. Department of Energy through contract DE-AC02-98CH10886 at Brookhaven National Laboratory, including Laboratory Directed R\&D funds. 


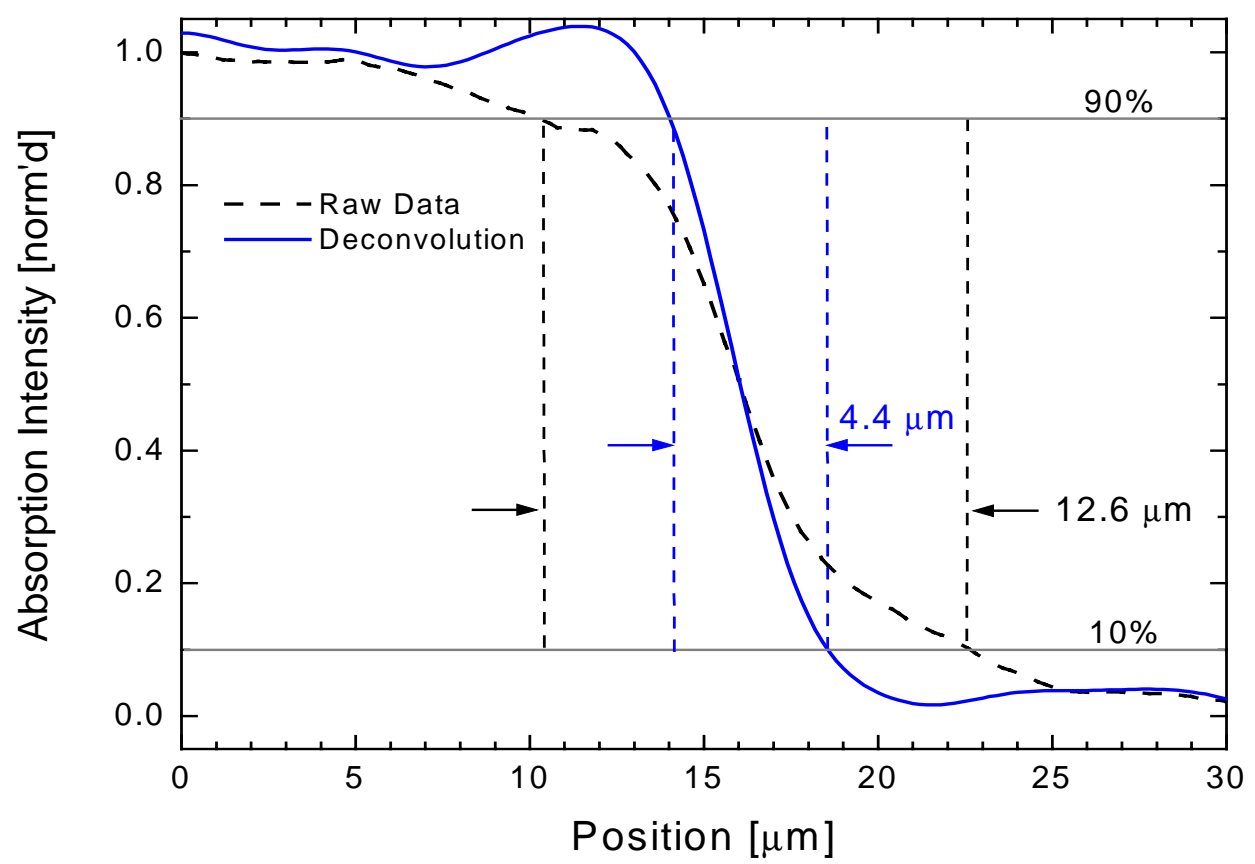

Fig. 1 Absorption as a function of position for $\lambda=6 \mu \mathrm{m}$ (dashed curve), determined from an IR microspectrometer scan across the edge of a polyamide photoresist layer. Diffraction blurs the nominally sharp edge to a width greater than $12 \mu \mathrm{m}$. Mathematical deconvolution (solid curve) yields an edge nearly 3 times sharper.

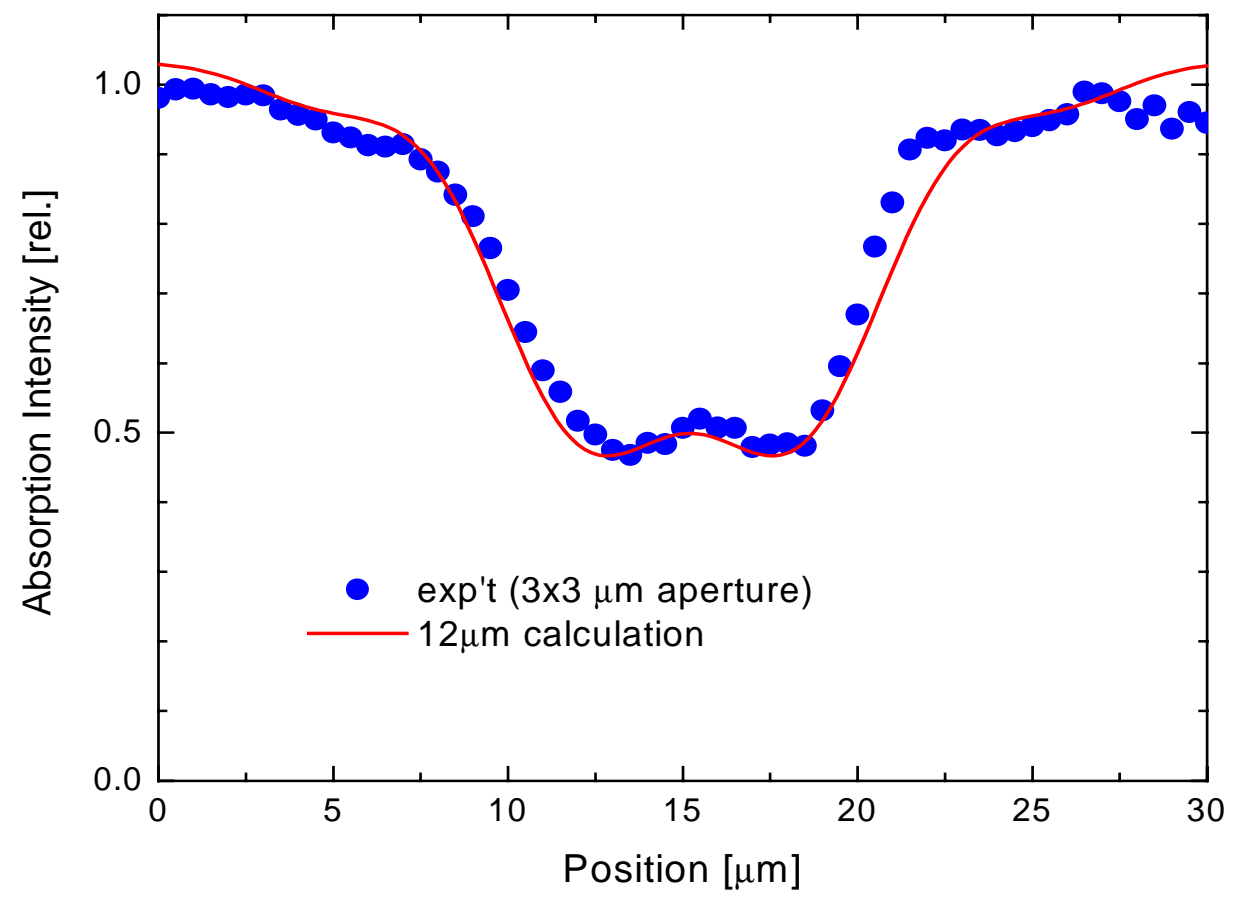

Fig. 2 The absorption profile (solid circles) at $\lambda=6 \mu \mathrm{m}$ from a scan across a photoresist layer with a $12 \mu \mathrm{m}$ diameter circular hole. The hole begins at $9 \mu \mathrm{m}$ and the far edge is reached at $21 \mu \mathrm{m}$. Also shown is the predicted profile assuming diffraction effects (solid line). Diffraction not only prevents the intensity from reaching zero inside the hole, it also leads to an artificial "bump" at the center. 